PROCEEDINGS OF THE

AMERICAN MATHEMATICAL SOCIETY

Volume 126, Number 10, October 1998, Pages 3109-3116

S $0002-9939(98) 04398-6$

\title{
THE INTERSECTION OF THREE SPHERES IN A SPHERE AND A NEW APPLICATION OF THE SATO-LEVINE INVARIANT
}

\author{
EIJI OGASA
}

(Communicated by Ronald A. Fintushel)

\begin{abstract}
Take transverse immersions $f: S_{1}^{4} \amalg S_{2}^{4} \amalg S_{3}^{4} \rightarrow S^{6}$ such that (1) $f \mid S_{i}^{4}$ is an embedding, (2) $f\left(S_{i}^{4}\right) \cap f\left(S_{j}^{4}\right) \neq \varnothing$ and $f\left(S_{i}^{4}\right) \cap f\left(S_{j}^{4}\right)$ is connected, and (3) $f\left(S_{1}^{4}\right) \cap f\left(S_{2}^{4}\right) \cap f\left(S_{3}^{4}\right)=\varnothing$. Then we obtain three surface-links $L_{i}=\left(f^{-1}\left(f\left(S_{i}^{4}\right) \cap f\left(S_{j}^{4}\right)\right), f^{-1}\left(f\left(S_{i}^{4}\right) \cap f\left(S_{k}^{4}\right)\right)\right)$ in $S_{i}^{4}$, where $(i, j, k)=(1,2,3)$, $(2,3,1),(3,1,2)$. We prove that, we have the equality $\beta\left(L_{1}\right)+\beta\left(L_{2}\right)+\beta\left(L_{3}\right)=$ 0 , where $\beta\left(L_{i}\right)$ is the Sato-Levine invariant of $L_{i}$, if all $L_{i}$ are semi-boundary links.
\end{abstract}

\section{INTRODUCTION AND MAIN RESULTS}

Take transverse immersions $f: S_{1}^{4} \amalg S_{2}^{4} \amalg S_{3}^{4} \rightarrow S^{6}$ such that (1) $f \mid S_{i}^{4}$ is an embedding, (2) $f\left(S_{i}^{4}\right) \cap f\left(S_{j}^{4}\right) \neq \varnothing$ and $f\left(S_{i}^{4}\right) \cap f\left(S_{j}^{4}\right)$ is connected, and $(3) f\left(S_{1}^{4}\right) \cap$ $f\left(S_{2}^{4}\right) \cap f\left(S_{3}^{4}\right)=\varnothing$. Then we obtain three surface-links

$$
L_{i}=\left(f^{-1}\left(f\left(S_{i}^{4}\right) \cap f\left(S_{j}^{4}\right)\right), f^{-1}\left(f\left(S_{i}^{4}\right) \cap f\left(S_{k}^{4}\right)\right)\right)
$$

in $S_{i}^{4}$, where $(i, j, k)=(1,2,3),(2,3,1),(3,1,2)$. An orientation is given to each naturally. In this paper, we discuss which ones we obtain.

In order to state our theorems, we need some definitions.

We work in the smooth category. $S_{i}^{4} \cap S_{j}^{4}$ is a closed orientable connected surface and is oriented naturally. Hereafter, a surface will always mean a closed oriented connected surface unless otherwise stated.

A surface- $\left(F_{1}, \ldots, F_{\mu}\right)$-link is a submanifold $L=\left(K_{1}, \ldots, K_{\mu}\right)$ of $S^{4}$ such that $K_{i}$ is diffeomorphic to the oriented surface $F_{i}$. If $\mu=1, L$ is called a surface- $F_{1}-k n o t$. A surface- $\left(F_{1}, F_{2}\right)$-link $L=\left(K_{1}, K_{2}\right)$ is called a semi-boundary link if $\left[K_{i}\right]=0$ $\in H_{2}\left(S^{4}-K_{j} ; \mathbb{Z}\right)(i \neq j)$ ([18]). A surface- $\left(F_{1}, F_{2}\right)$-link $L=\left(K_{1}, K_{2}\right)$ is called a boundary link if there exist Seifert hypersurfaces $V_{i}$ for $K_{i}(i=1,2)$ such that $V_{1} \cap V_{2}=\varnothing$. A surface- $\left(F_{1}, F_{2}\right)$-link $\left(K_{1}, K_{2}\right)$ is called a split link if there exist 4-balls $B_{1}^{4}$ and $B_{2}^{4}$ in $S^{4}$ such that $B_{1}^{4} \cap B_{2}^{4}=\varnothing$ and $K_{i} \subset B_{i}^{4}$.

Received by the editors July 23, 1996 and, in revised form, February 26, 1997.

1991 Mathematics Subject Classification. Primary 57M25, 57Q45.

Key words and phrases. Surface-knot, surface-link, spin cobordism group, the Sato-Levine invariant, realizable triple of surface-links.

This research was partially suppported by Research Fellowships of the Promotion of Science for Young Scientists.

(C)1998 American Mathematical Society 
Definition. $\left(L_{1}, L_{2}, L_{3}\right)$ is called a triple of surface-links if $L_{1}$ is a $\left(F_{12}, F_{13}\right)$ link, $L_{2}$ is a $\left(F_{23}, F_{21}\right)$-link, $L_{3}$ is a $\left(F_{31}, F_{32}\right)$-link, and $F_{i j}$ is diffeomorphic to $F_{j i}((i, j)=(1,2),(2,3),(3,1))$.

Definition. A triple of surface-links $\left(L_{1}, L_{2}, L_{3}\right)$ is said to be realizable if there exists a transverse immersion $f: S_{1}^{4} \amalg S_{2}^{4} \amalg S_{3}^{4} \rightarrow S^{6}$ such that (1) $f \mid S_{i}^{4}$ is an embedding $(i=1,2,3)$, (2) $\left(f^{-1}\left(f\left(S_{i}^{4}\right) \cap f\left(S_{j}^{4}\right)\right), f^{-1}\left(f\left(S_{i}^{4}\right) \cap f\left(S_{k}^{4}\right)\right)\right)$ in $S_{i}^{4}$ is $L_{i}=\left(K_{i j}, K_{i k}\right) \quad((i, j, k)=(1,2,3),(2,3,1),(3,1,2))$, and $(3) f\left(S_{1}^{4}\right) \cap f\left(S_{2}^{4}\right) \cap f\left(S_{3}^{4}\right)$ $=\varnothing$.

We state the main theorem.

Theorem 1.1. Let $L_{1}, L_{2}$ and $L_{3}$ be semi-boundary surface-links. Let $\left(L_{1}, L_{2}, L_{3}\right)$ be a triple of surface-links. Suppose the triple of surface-links $\left(L_{1}, L_{2}, L_{3}\right)$ is realizable. Then we have the equality

$$
\beta\left(L_{1}\right)+\beta\left(L_{2}\right)+\beta\left(L_{3}\right)=0,
$$

where $\beta\left(L_{i}\right)$ is the Sato-Levine invariant of $L_{i}$.

We review the Sato-Levine invariants in $\S 2$. Since there exists a triple of surfacelinks $\left(L_{1}, L_{2}, L_{3}\right)$ such that $\beta\left(L_{1}\right)=\beta\left(L_{2}\right)=0$ and $\beta\left(L_{3}\right)=1$ (see $\S 2$ ), we have:

Corollary 1.2. Not all triple of surface-links are realizable.

We prove:

Theorem 1.3. There exists a realizable triple of surface-links $\left(L_{1}, L_{2}, L_{3}\right)$ such that $\beta\left(L_{1}\right)=1, \beta\left(L_{2}\right)=1$, and $\beta\left(L_{3}\right)=0$.

We prove the following sufficient conditions for the realization.

Theorem 1.4. Let $L_{1}, L_{2}$ and $L_{3}$ be split surface-links. Let $\left(L_{1}, L_{2}, L_{3}\right)$ be a triple of surface-links. Then the triple of surface-links $\left(L_{1}, L_{2}, L_{3}\right)$ is realizable.

Theorem 1.5. Suppose $L_{i}$ are $\left(S^{2}, S^{2}\right)$-links and $L_{i}$ are slice links $(i=1,2,3)$. Then the triple of surface-links $\left(L_{1}, L_{2}, L_{3}\right)$ is realizable.

We give problems.

Problem 1.6. (1) Determine the realizable triple of surface-links. (2) Is the inverse of Theorem 1.1 valid? (3) Let $L_{1}, L_{2}$ and $L_{3}$ be $\left(S^{2}, S^{2}\right)$-links. Then is the triple of surface-links $\left(L_{1}, L_{2}, L_{3}\right)$ realizable?

Note. (i) Using a result of [15] (see §2), one can show Problem 1.6.(3) follows from Problem 1.6.(2). (ii) By Theorem 1.5, if the answer to Problem 1.6.(3) is negative, then the answer to an outstanding problem: "Is every $\left(S^{2}, S^{2}\right)$-link slice?" is negative. (Refer to [5], [6], and [12] for the slice ploblem.)

This paper is organized as follows. In $\S 2$ we review the Sato-Levine invariant. In $\S 3$ we prove Theorem 1.1. In $\S 4$ we prove Theorem 1.3. In $\S 5$ we prove Theorem 1.4. In $\S 6$ we prove Theorem 1.5.

\section{The Sato-Levine invariant and Spin COBORDism}

The Sato-Levine invariant is defined by Sato (in [18]) and Levine (unpublished) independently. It is easy to prove that the following definition is equivalent to theirs. 
Definition. Let $L=\left(K_{1}, K_{2}\right)$ be a semi-boundary surface- $\left(F_{1}, F_{2}\right)$-link. Then there exist Seifert hypersurfaces $V_{i}$ for $K_{i}(i=1,2)$ such that $V_{i} \cap K_{j}=\varnothing(i \neq j)$. Let $v_{i}$ be the oriented normal bundle of $V_{i}$ in $S^{4}$. Let $F$ be the oriented closed surface $V_{1} \cap V_{2}$. F need not be connected. Then the congruence $\left.T S^{4}\right|_{F} \cong T F \oplus$ $\left.\left.v_{1}\right|_{F} \oplus v_{2}\right|_{F}$ induces a spin structure $\sigma$ on $F$. We define the Sato-Levine invariant $\beta(L)$ of $L$ so that $\beta(L)=[(F, \sigma)] \in \Omega_{2}^{\text {spin }} \cong \mathbb{Z}_{2}$ for $L$. We call $(F, \sigma)$ a special surface for $L$.

By [17] and [18] the following holds.

Theorem ([17] and [18]). Let $F_{1}$ be an oriented closed connected surface not diffeomorphic to the 2-sphere. Let $F_{2}$ be an arbitrary oriented closed connected surface. Then there exists a semi-boundary $\left(F_{1}, F_{2}\right)$-link whose Sato-Levine invariant is one.

In [15] Orr proved the following.

Theorem ([15]). The Sato-Levine invariant of an arbitrary $\left(S^{2}, S^{2}\right)$-link is zero.

The Sato-Levine invariant and its generalization are studied in [1], [2], [3], [4], [7], [8], [10], [11], [16], [19], [20], p.103 of [21], etc. [2] says that the Sato-Levine invariant is connected with [9].

\section{The Proof of Theorem 1.1}

Let $L_{1}=\left(K_{12}, K_{13}\right), L_{2}=\left(K_{23}, K_{21}\right)$, and $L_{3}=\left(K_{31}, K_{32}\right)$. Let $f: S_{1}^{4} \amalg S_{2}^{4} \amalg$ $S_{3}^{4} \leftrightarrow S^{6}$ be an immersion to realize $\left(L_{1}, L_{2}, L_{3}\right)$. We abbreviate $f\left(S_{i}^{4}\right)$ to $S_{i}^{4}$. We first prove:

Claim. There exist Seifert hypersurfaces $A_{i}$ for $S_{i}^{4}(i=1,2,3)$ such that $A_{1} \cap S_{2}^{4} \cap$ $S_{3}^{4}=\varnothing, A_{2} \cap S_{3}^{4} \cap S_{1}^{4}=\varnothing$, and $A_{3} \cap S_{1}^{4} \cap S_{2}^{4}=\varnothing$.

Proof. Let $S_{2}^{4} \times D^{2}$ be a tubular neighborhood of $S_{2}^{4}$ in $S^{6}$. Put $D^{2}=\left\{(x, y) \mid x^{2}+\right.$ $\left.y^{2} \leqq 0\right\}$. Then $S_{2}^{4}=S_{2}^{4} \times\{(0,0)\}$. Put $I=\{(x, y) \mid 0 \leqq x \leqq 1, y=0\}$. We can regard $S_{2}^{4} \times D^{2}$ as the result of rotating $S_{2}^{4} \times I$ around the axis $S_{2}^{4}$.

Put $M=\left(S_{2}^{4} \times I\right) \cap S_{1}^{4}$. As we rotate $S_{2}^{4} \times I$ as above, we rotate $M$ as well. The result is $\left(S_{2}^{4} \times D^{2}\right) \cap S_{1}^{4}$.

Take a Seifert hypersurface $A_{1}^{\prime}$ for $S_{1}^{4}$. Then $A_{1}^{\prime} \cap S_{2}^{4}$ in $S_{2}^{4}$ is a Seifert hypersurface $V_{21}^{\prime}$ for $K_{21}$. We can suppose that $A_{1}^{\prime} \cap\left(S_{2}^{4} \times p\right)$ in $S_{2}^{4} \times p$ is the submanifold $V_{21}^{\prime}$ for each $p \in D^{2}$.

Since $L_{2}=\left(K_{23}, K_{21}\right)$ is a semi-boundary link, there is a Seifert hypersurface $V_{21}$ for $K_{21}$ such that $V_{21} \cap K_{23}=\varnothing$. Then there exists a compact oriented 4-manifold $W$ in $S_{2}^{4} \times I$ with the following properties.

(1) $W \cap S_{2}^{4}$ in $S_{2}^{4}$ is the submanifold $V_{21}$.

(2) $W \cap\left(S_{2}^{4} \times\{(1,0)\}\right)$ in $\left(S_{2}^{4} \times\{(1,0)\}\right)$ is the submanifold $V_{21}^{\prime}$.

(3) $(\partial W)-V_{21}^{2}-V_{21}^{\prime 2}$ is $M$.

When we rotate $S_{2}^{4} \times I$ as above, we rotate $W$ together. Let $P$ denote what is made from $W$. Note that $\partial P=\partial\left(\overline{A_{1}^{\prime} \cap\left(S_{2}^{4} \times D^{2}\right)}\right)=\partial\left(\overline{A_{1}^{\prime}-\left(A_{1}^{\prime} \cap\left(S_{2}^{4} \times D^{2}\right)\right)}\right)$ $-S_{1}^{4}$. Let $A_{1}=\overline{A_{1}^{\prime}-A_{1}^{\prime} \cap\left(S_{2}^{4} \times D^{2}\right)} \cup P$.

Then $A_{1} \cap S_{2}^{4} \cap S_{3}^{4}=V_{21} \cap K_{23}=\varnothing$. Note that, when we modify $A_{1}^{\prime}$ to obtain $A_{1}$, we don't change $f$.

Replace $(1,2,3)$ with $(2,3,1)$ (resp. $(3,1,2))$ in the above proof. Then we obtain $A_{2}$ (resp. $A_{3}$ ). We now obtain $A_{1}, A_{2}$ and $A_{3}$ so that we keep the immersion $f$. This completes the proof. 
Put $X=A_{1} \cap A_{2} \cap A_{2}$. Put $F_{i}=(\partial X) \cap S_{i}^{4}$. Then $\partial X=F_{1} \amalg F_{2} \amalg F_{3}$. By using $A_{1}, A_{2}, A_{3}$ and $S^{6}$, we give $F_{i}$ (resp. $W$ ) a spin structure $\sigma_{i}$ (resp. $\tau$ ). Of course $\partial(X, \tau)=\coprod_{i=1}^{3}\left(F_{i}, \sigma_{i}\right)$. Then $\left(F_{i}, \sigma_{i}\right)$ is a special surface for $L_{i}$. Therefore, $\sum_{i} \beta\left(L_{i}\right)=\sum_{i}\left[\left(F_{i}, \sigma_{i}\right)\right]=[\partial(X, \tau)]=0 \in \Omega_{2}^{\text {Spin }}$.

\section{The Proof of Theorem 1.3}

Let $L_{1}=\left(K_{12}, K_{13}\right)$ be the $\left(T^{2}, S^{2}\right)$-link in [17]. Let $L_{2}=\left(K_{23}, K_{21}\right)$ be the $\left(S^{2}, T^{2}\right)$-link obtained by changing the order of $L_{1}$. Let $L_{3}=\left(K_{31}, K_{32}\right)$ be the trivial $\left(S^{2}, S^{2}\right)$-link. Note $\beta\left(L_{1}\right)=\beta\left(L_{2}\right)=1$ and $\beta\left(L_{3}\right)=0$. It suffices to prove that the triple of surface-links $\left(L_{1}, L_{2}, L_{3}\right)$ is realizable.

$\left(K_{1}, K_{2}\right)$ is called a pair of surface-F-knots if both $K_{1}$ and $K_{2}$ are $F$-knots. A pair of $F$-knots $\left(K_{1}, K_{2}\right)$ is said to be realizable if there exists a transverese immersion $f: S_{1}^{4} \amalg S_{2}^{4} \uparrow S^{6}$ such that (1) $f \mid S_{i}^{4}$ is an embedding $(i=1,2)$, and (2) $f^{-1}\left(f\left(S_{1}^{4}\right) \cap f\left(S_{2}^{4}\right)\right)$ in $S_{i}^{4}$ is $K_{i}(i=1,2)$.

We prove:

Proposition 4.1. Let $K$ be a surface-knot. Then the pair of surface-knots $(K, K)$ is realizable.

Take an embedding $f: S_{1}^{4} \amalg S_{2}^{4} \hookrightarrow S^{6}$. There exists a chart $U$ of $S^{6}$ such that

(1) $\phi: U \cong \mathbb{R}^{4} \times\{(u, v) \mid u, v \in \mathbb{R}\} \cong \mathbb{R}^{4} \times \mathbb{R}_{u} \times \mathbb{R}_{v}$, and

$(2) U \cap f\left(S_{1}^{4}\right)=\mathbb{R}^{4} \times\{(u, v) \mid u=0, v=0\}$. Call it $\mathbb{R}_{1}^{4} . U \cap f\left(S_{2}^{4}\right)=\mathbb{R}^{4} \times\{(u, v) \mid u=$ $1, v=0\}$. Call it $\mathbb{R}_{2}^{4}$.

We prove Lemma 4.2. Obviously it induces Proposition 4.1.

Lemma 4.2. There exists a transverse immersion $g: S_{1}^{4} \amalg S_{2}^{4} \uparrow S^{6}$ to realize the pair of surface-knots $(K, K)$ with the following properties.

(1) $g\left|S_{2}^{4}=f\right| S_{2}^{4}$.

(2) $g\left(S_{2}^{4}\right) \cap \mathbb{R}^{4} \times\{u=0\} \times \mathbb{R}_{v}=g\left(S_{2}^{4}\right) \cap \mathbb{R}^{4} \times\{u=0\} \times\{v=0\}$.

(3) $g \mid S_{1}^{4}$ is isotopic to $f \mid S_{1}^{4}$.

We modify the embedding $f$ to obtain an immersion $g$.

Take any Seifert hypersurface $V$ for $K$ in $\mathbb{R}_{1}^{4}$. Let $N(V)=V \times\{t \mid-1 \leqq t \leqq 1\}$ be a tubular neighborhood of $V$ in $\mathbb{R}_{1}^{4}$. We define the subset $E$ of $N(V) \times \mathbb{R}_{u} \times \mathbb{R}_{v}$ $=\{(p, t, u, v) \mid p \in V,-1 \leqq t \leqq 1, u \in \mathbb{R}, v \in \mathbb{R}\}$ so that

$$
E=\left\{(p, t, u, v) \mid p \in V, 0 \leqq u \leqq \frac{\pi}{2}, t=k \cdot \cos u, v=k \cdot \sin u,-1 \leqq k \leqq 1\right\} .
$$

Put $X=\overline{(\partial E)-N(V)}$ and $Y=\overline{f\left(S_{1}^{4}\right)-N(V)}$. Then $\partial X=\partial Y=\partial N(V)$. Put $\Sigma=X \cup Y$. Then $\Sigma$ is an embedded 4-sphere. We define $g \mid S_{1}^{4}$ so that $g\left(S_{1}^{4}\right)=\Sigma$. This completes the proof of Lemma 4.2 and therefore Proposition 4.1.

Note. See Figure 4.1. We draw a lower dimensional analogue. There, we replace $\mathbb{R}^{4} \times \mathbb{R}_{u} \times \mathbb{R}_{v}$ with $\mathbb{R}^{2} \times \mathbb{R}_{u} \times \mathbb{R}_{v}$.

By the definition of $L_{i}$, the $T^{2}$-knots $K_{12}$ and $K_{21}$ are equivalent. Therefore there is an immersion $g: S_{1}^{4} \coprod S_{2}^{4} \rightarrow S^{6}$ to realize the pair of $T^{2}$-knots $\left(K_{12}, K_{21}\right)$.

We prove the following Lemma 4.3. Obviously Lemma 4.3 induce Theorem 1.3.

Lemma 4.3. There exists a transverse immersion $h: S_{1}^{4} \amalg S_{2}^{4} \amalg S_{3}^{4} \hookrightarrow S^{6}$ to realize $\left(L_{1}, L_{2}, L_{3}\right)$ with the following properties.

(1) $\left.h\right|_{S_{1}^{4} \amalg S_{2}^{4}}=g$,

(2) $h\left(S_{3}^{4}\right) \subset U . h\left(S_{3}^{4}\right)$ is the trivial 3-knot. 

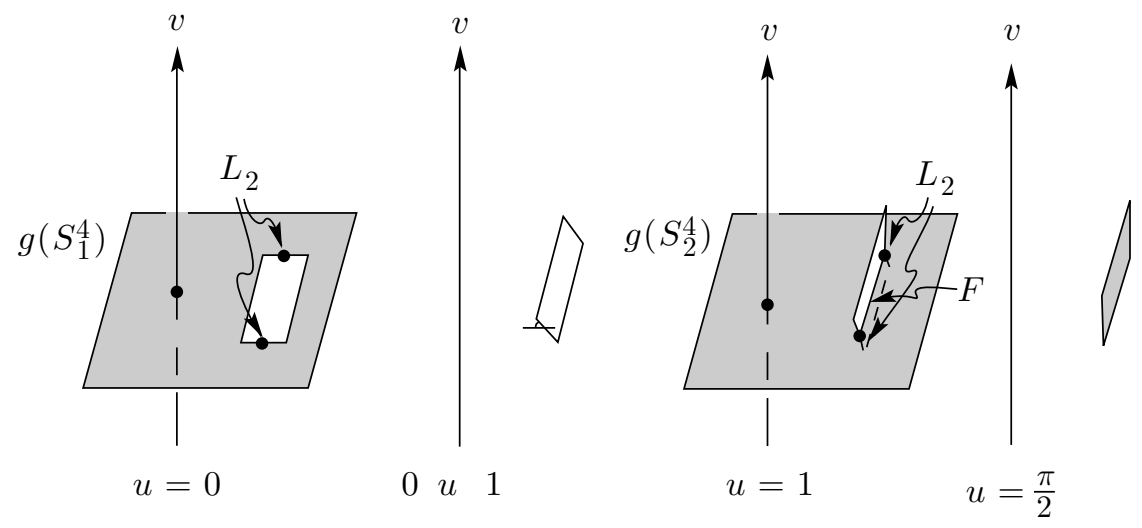

Figure 4.1

Proof. We modify the immersion $g$ to obtain an immersion $h$.

Take $K_{13}$ (resp. $K_{23}$ ) in $\mathbb{R}_{1}^{4}$ (resp. $\mathbb{R}_{2}^{4}$ ). There is a Seifert hypersurface $V_{12}$ for $K_{12}$ so that $V_{12} \cap K_{13}=\varnothing$. Take $V_{12}$ as a Seifert hypersurface used in the proof of Lemma 4.2. Recall $V_{12}$ and $K_{13}$ are in $\mathbb{R}_{1}^{4}$.

Recall $K_{13}$ and $K_{23}$ are the trivial $S^{2}$-knots. Take a 3-ball $B_{13}^{3}$ (resp. $B_{23}^{3}$ ) which bounds $K_{13}$ (resp. $K_{23}$ ) in $\mathbb{R}_{1}^{4}\left(\right.$ resp. $\mathbb{R}_{2}^{4}$ ). Note that $B_{13}^{3}$ is not included in $g\left(S_{1}^{4}\right)$.

Take the 5-ball $B^{5}=\left\{(q, u, v) \mid q \in B^{3},-1 \leqq u \leqq 2,-2 \leqq v \leqq 2\right\}$ in $U$. Suppose $B^{5} \cap \mathbb{R}_{1}^{4}=B_{13}^{3}$ and $B^{5} \cap \mathbb{R}_{2}^{4}=B_{23}^{3}$. Then

$$
\left(\partial B^{5}\right) \cap S_{1}^{4} \cap S_{2}^{4}=\varnothing .
$$

Define $h \mid S_{3}^{4}$ so that $h\left(S_{3}^{4}\right)=\partial B^{5}$.

This completes the proof of Lemma 4.3 and hence Theorem 1.3.

\section{The proof of Theorem 1.4}

\section{AND A RELATION BETWEEN KNOT COBORDISM}

AND THE REALIZATION OF A PAIR OF KNOTS

Surface- $F$-knots $K_{0}$ and $K_{1}$ are said to be cobordant or concordant if there is a smooth submanifold $W$ of $S^{4} \times[0,1]$, which meets the boundary transversely in $\partial W$, is diffeomorphic to $F \times[0,1]$ and meets $S^{4} \times\{i\}$ in $K_{i}(i=0,1)$.

We prove the following although it may be folklore.

Theorem 5.1. Let $F$ be a closed connected oriented surface. Then arbitrary Fknots $K_{0}$ and $K_{1}$ are cobordant.

Proof. Let $L$ be a split surface-link with components $K_{0}$ and $-K_{1}$. It suffices to prove:

Claim. There exists a submanifold of $S^{4}$ which is diffeomorphic to $F \times[0,1]$ such that $F \times[0,1]$ intersects with $\partial B^{5}$ transversely, $F \times[0,1] \cap \partial B^{5}=F \times\{0\} \amalg F \times\{1\}$, and $(F \times\{0\}, F \times\{1\})$ in $S^{4}=\partial B^{5}$ is $L$.

Let $V$ be a connected Seifert hypersurface for $L$. A spin structure on $V$ is induced from the unique one on $S^{4}$. A spin structure on $\partial V$ is induced from the one on $V$. 
Make a closed spin 3-manifold $W=V \cup(F \times[0,1])$ so that the spin structure on $V$ extend to the one on $W$. Note $W$ is not a submanifold of $S^{4}$. Since $\Omega_{3}^{\text {spin }}=0$, there exists a spin 4 -manifold $X$ which $W$ spin-bounds. Since $V$ and $F \times[0,1]$ are connected, we can take a handle decomposition

$$
X=(V \times[0,1]) \cup\left(4 \text {-dimensional 2-handles } h^{2}\right) \cup\{(F \times[0,1]) \times[0,1]\} .
$$

Take $V \times[0,1]$ in $S^{4} \times[0,1]$ so that $V \times\{t\}$ is in $S^{4} \times\{t\}$. Attach the handles $h^{2}$ to $V \times\{1\} \subset S^{4} \times\{1\}$. Then we can attach the 5-dimensional 2-handles $\bar{h}^{2}=h^{2} \times[-1,1]$ to $S^{4} \times\{1\}$ naturally. Let $Y=S^{4} \times[0,1] \cup$ (the 5-dimensioanl 2handles $\bar{h}^{2}$ ). Since the attaching maps of $\bar{h}^{2}$ are spin preserving diffeomorphisms, $Y$ is diffeomorphic to $\left(\natural^{*} S^{2} \times B^{3}\right)-($ the 5 -ball). $\partial Y$ is a disjoint union of the standard 4 -sphere $S_{0}^{4}$ and $\left(\sharp^{*} S^{2} \times S^{2}\right)$. Hence $Y$ is embedded in $B^{5}$ so that $S_{0}^{4}$ coincides with $\partial B^{5}$.

Therefore $F \times[0,1] \subset W \subset B^{5}$ and the submanifold $F \times[0,1]$ satisfies the condition in the claim. This completes the proof.

It is easy to prove that Theorem 1.4 is equivalent to the following Theorem 5.2. We prove:

Theorem 5.2. Let $F$ be a closed connected oriented surface. If $F$-knots $K$ and $K^{\prime}$ are cobordant, the pair of $F$-knots $\left(K, K^{\prime}\right)$ is realizable.

Proof. By Proposition 4.1, the pair of $F$-knots $\left(K, K^{\prime}\right)$ is realizable. Hence it suffices to prove:

Claim. Suppose that a pair of $F$-knots $\left(K_{1}, K_{2}\right)$ is realizable. Suppose that $K_{2}$ is cobordant to $K_{3}$. Then $\left(K_{1}, K_{3}\right)$ is realizable.

Proof. Let $f: S_{1}^{4} \amalg S_{2}^{4} \rightarrow S^{6}$ be an immersion to realize $\left(K_{1}, K_{2}\right)$. We construct an immersion $\widetilde{f}: S_{1}^{4} \amalg S_{2}^{4} \leftrightarrow S^{6}$ to realize $\left(K_{1}, K_{3}\right)$ as follows. Put $\widetilde{f}\left|S_{2}^{4}=f\right| S_{2}^{4}$.

Let $f\left(S_{2}^{4}\right) \times D^{2}$ be a tubular neighborhood of $S_{2}^{4}$ in $S^{6}$. Put $D^{2}=\left\{(x, y) \mid x^{2}+\right.$ $\left.y^{2} \leqq 0\right\}$. Then $f\left(S_{2}^{4}\right)=S_{2}^{4} \times\{(0,0)\}$. Put $I=\{(x, y) \mid 0 \leqq x \leqq 1, y=0\}$. We can regard $f\left(S_{2}^{4}\right) \times D^{2}$ as what is obtained by rotating $f\left(S_{2}^{4}\right) \times I$ around $f\left(S_{2}^{4}\right)$ as the axis.

Put $M=\left(f\left(S_{2}^{4}\right) \times I\right) \cap f\left(S_{1}^{4}\right)$. We can regard $\left(f\left(S_{2}^{4}\right) \times D^{2}\right) \cap f\left(S_{1}^{4}\right)$ as what is made from $M$ as follows: When we rotate $\left(f\left(S_{2}^{4}\right) \times I\right)$ as above, we rotate $M$ together. What is made from $M$ is $\left(f\left(S_{2}^{4}\right) \times D^{2}\right) \cap f\left(S_{1}^{4}\right)$.

We can suppose that $\left\{f\left(S_{2}^{4} \times p\right)\right\} \cap f\left(S_{1}^{4}\right)$ in $f\left(S_{2}^{4}\right) \times p$ is $K_{2}$ for each $p \in D^{2}$.

Since $K_{2}$ and $K_{3}$ are cobordant, there is a compact oriented 3-manifold $P$ in $f\left(S_{2}^{4}\right) \times I$ with the following properties. (1) $P \cong F \times[0,1]$. (2) $P$ intersects $f\left(S_{2}^{4}\right) \times \partial I$ transversely. $P \cap f\left(S_{2}^{4}\right)$ in $f\left(S_{2}^{4}\right)$ is $K_{3} . P \cap\left[f\left(S_{2}^{4}\right) \times\{(1,0)\}\right]$ in $f\left(S_{2}^{4}\right) \times\{(1,0)\}$ is $K_{2}$.

When we rotate $f\left(S_{2}^{4}\right) \times I$ as above, rotate $P$ together. Let $Q$ denote what is made from $P$.

Note that

$$
\left.\left.\partial Q=\partial \overline{f\left(S_{1}^{4}\right) \cap\left(f\left(S_{2}^{4}\right) \times D^{2}\right)}\right)=\partial \overline{f\left(S_{1}^{4}\right)-\left(f\left(S_{1}^{4}\right) \cap\left(f\left(S_{2}^{4}\right) \times D^{2}\right)\right.}\right) .
$$

Then $R=\overline{f\left(S_{1}^{4}\right)-\left(f\left(S_{1}^{4}\right) \cap\left(f\left(S_{2}^{4}\right) \times D^{2}\right)\right)} \cup Q$ is a 4 -sphere embedded in $S^{6}$. Put $\widetilde{f}\left(S_{2}^{4}\right)=R$. This completes the proof. 


\section{The PRoOF of Theorem 1.5}

It is easy to prove that it suffices to prove:

Proposition. Let $L=\left(K_{1}, K_{2}\right)$ be a $\left(S^{2}, S^{2}\right)$-link and a slice link. Then there exists three 4-spheres $S_{1}^{4}, S_{2}^{4}$, and $S^{4}$ embedded in $S^{6}$ with the following properties.

(1) $S_{1}^{4} \cap S_{2}^{4}=\varnothing$.

(2) $\left(S_{1}^{4} \cap S^{4}, S_{2}^{4} \cap S^{4}\right)$ in $S^{4}$ is $L$.

Proof. Let $S^{4} \times D^{2}$ denote a tubular neighborhood of $S^{4}$ in $S^{6}$. Put $D^{2}=$ $\left\{(x, y) \mid x^{2}+y^{2} \leqq 0\right\}$. Then $S^{4}=S^{4} \times\{(0,0)\}$. Put $I=\{(x, y) \mid 0 \leqq x \leqq 1, y=0\}$. We can regard $S^{4} \times D^{2}$ as the result of rotating $S^{4} \times I$ around the axis $S^{4}$.

Since the 2-link $L$ is slice, there exists two 3 -discs $D_{1}^{3}$ and $D_{2}^{3}$ in $S^{4} \times I$ with the following properties. (1) $D_{1}^{3} \cap D_{2}^{3}=\varnothing$. (2) $D_{i}^{3}$ intersects $S^{4}$ transversely. $D_{i}^{3}$ $\cap S^{4}=\partial D_{i}^{3}$. (3) $\left(\partial D_{1}^{3}, \partial D_{2}^{3}\right)$ in $S^{4}$ is the 2-link $L$.

When we rotate $S^{4} \times I$ as above, we rotate $D_{1}^{3} \amalg D_{2}^{3}$ together. This gives 4 -spheres $S_{1}^{4}$ and $S_{2}^{4}$ embedded in $S^{6}$. This completes the proof.

\section{Acknowledgement}

The author would like to thank Dr. Yoshitake for his question on the author's paper [14] which is a motivation of this paper. The author would also like to thank Prof. Takashi Inaba for his question on Problem 1.6(2) which is a motivation of Theorem 1.3.

\section{REFERENCES}

[1] P.Akhmetiev and A.Ruzmaikin, A fourth-order topological invariant of magnetic or voltex lines, J. Geom. Phys. 15 (1995), 95-101. MR 96b:57003

[2] T. D. Cochran, Geometric invariants of link cobordism, Comment.Math.Helv. 60 (1985), 291-311. MR 87f:57021

[3] T. D. Cochran, Link concordance invariants and homotopy theory, Invent.Math. 90 (1987), 635-645. MR 89f:57033

[4] T. D. Cochran, Derivatives of links: Milnor's concordance invariants and Massey's products, Mem.Amer.Math.Soc. 427 (1990). MR 91c:57005

[5] T.D.Cochran and K. E. Orr, Not all links are concordant to boundary links, Ann. of Math. 138 (1993), 519-554. MR 95c:57042

[6] P. Gilmer and C. Livingston, The Casson-Gordon invariant and link concordance, Topology 31 (1992), 475-492. MR 93h:57037

[7] P. Gilmer, Classical knot and link concordance, Comment.Math.Helv. 68 (1993), 1-19. MR 94c:57007

[8] P. Kirk and C. Livingston, Vassiliev invariants of two component links and the CassonWalker invariants, Topology 36 (1997), 1333-1353. CMP 97:13

[9] S. Kojima and M. Yamasaki, Some new invariants of links, Invent.Math. 54 (1979), 213-228. MR 81b:57004

[10] J.Levine, Link concordance and algebraic closure of groups, Comment.Math.Helv. 64 (1989), 236-255. MR 91a:57016

[11] J.Levine, Link concordance and algebraic closure II, Invent. Math. 96 (1989), 571-592. MR 91g:57007

[12] J.Levine, Link invariants via the eta-invariant, Comment.Math.Helv. 69 (1994), 82-119. MR 95a:57009

[13] J.Levine, W.Mio, and K. Orr, Links with vanishing homotopy invariants, Comm. Pure \& Applied Math. XLVI (1993), 213-220. MR 94e:57036

[14] E. Ogasa, On the intersection of spheres in a sphere I, II, Tokyo University preprint (1995).

[15] K. E. Orr, New link invariants and applications, Comment.Math.Helv. 62 (1987), 542-560. MR 89a: 57029 
[16] K. E. Orr, Link concordance invariants and Massey products, Topology (1991). MR 93a:57025

[17] D. Ruberman, Concordance of links in $S^{4}$, Contmp.Math. 35 (1984), 481-483. MR 86g:57017

[18] N. Sato, Cobordisms of semi-boundary links, Topology Appl. 18 (1984), 225-234. MR 86d:57010

[19] M. Saito, On the unoriented Sato-Levine invariant, J. Knot Theory Ramifications 2 (1993), 335-358. MR 94h:57017

[20] M. Saito, A note on cobordism of surface links in $S^{4}$, Proc.Amer.Math.Soc. 111 (1991), 883-887. MR 92a:57027

[21] K.Walker, An extension of Casson's invariant, Princeton Univ.Press (1992). MR 93e:57031

Department of Mathematical Sciences, University of Tokyo, Komaba, Tokyo 153, JAPAN

E-mail address: i33992@m-unix.cc.u-tokyo.ac.jp 